\title{
Breakdown of order in a self-organised barrier discharge
}

\author{
R. Wild ${ }^{\mathrm{a}}$ and L. Stollenwerk \\ University of Greifswald, Institute of Physics, Felix-Hausdorff Str. 6, 17489 Greifswald, Germany
}

Received 2 April 2012 / Received in final form 4 June 2012

Published online 28 August 2012

(C) The Author(s) 2012. This article is published with open access at Springerlink.com

\begin{abstract}
In this work, we investigate the transition of a hexagonal patterned self-organised barrier discharge into a stochastic distributed pattern during voltage reduction. The techniques of 2D Fourier transformation and, for the first time, the triple correlation function are used. Introducing measures for the angular and radial order it is possible to observe a supercritical bifurcation scenario for the decaying order. A chronological sequence of an angular and a radial breakdown of the pattern has been revealed. Possible limits of reproducibility and dependence on the frequency of the applied voltage are estimated.
\end{abstract}

\section{Introduction}

Barrier discharges (BD) are gas discharge systems whose electrodes confine a discharge gap that hosts at least one dielectric barrier. The resulting capacitance in the electric circuit allows for an operation solely in AC mode. This particular plasma source permits applications such as ozone generation, surface modification, and plasma display panels [1-3]. Recent investigations even deal with the application in the medical field $[4,5]$.

Laterally extended systems, i.e. systems where the lateral extension is much larger than the discharge gap, have been found to produce self-organised structures. In this case, the discharge breaks up into a number of filaments that may show a collective behaviour yielding higher ordered structures. That way, the structures are observed to be e.g. hexagonal [6,7], squared [8], or an arrangement of concentric rings $[9,10]$. Further, it has been observed that it is possible to change the pattern by some variation of an operation parameter, such as the applied voltage or frequency $[11,12]$.

In this work, we investigate the transition process from a hexagonal pattern to a random arrangement of the filaments that occurs during the reduction of the applied voltage amplitude. The process is then investigated using 2D Fourier transformation and the triple correlation function. For both of these tools, a characteristic feature of the hexagonal pattern is used to define a measure for the hexagonal order. That way, the bifurcation behaviour can be investigated under parameter variation.

This contribution continues with a description of the experimental setup (Sect. 2) and a brief introduction to the triple correlation function (Sect. 3). The result section contains the discussion of the observed phenomenon

\footnotetext{
a e-mail: wild@physik.uni-greifswald.de
}

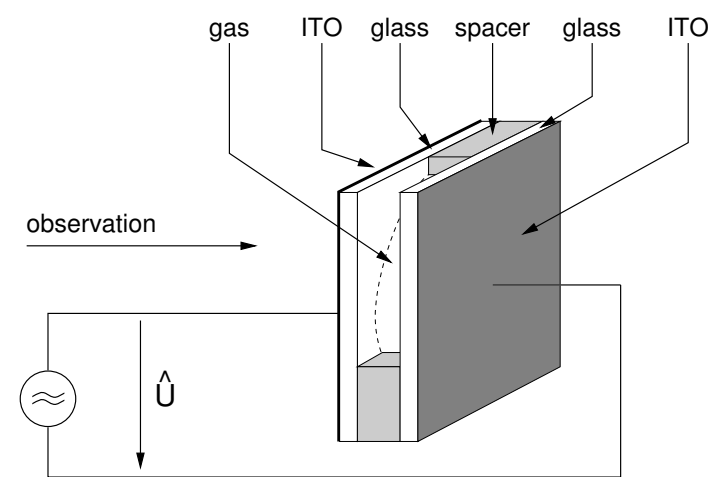

Fig. 1. (Color online) Schematic of the discharge cell setup. The cell consists of two glass layers with a thickness of $0.7 \mathrm{~mm}$ each and at a distance of $0.5 \mathrm{~mm}$ to one another. Both are ITO covered at the outer side and have been roughened at the gap side.

(Sect. 4) as well aspects of its reproducibility. Section 5 concludes this contribution.

\section{Experimental setup}

A schematic of the discharge cell is shown in Figure 1. It consists of two glass plates with a thickness of $0.7 \mathrm{~mm}$ each at a distance of $0.5 \mathrm{~mm}$ to each other. Both are covered with an ITO (indium tin oxide) layer on the outside acting as an electrode. Its optical transparency and electrical conductivity permits an observation of the discharge along current direction. On the gap side, both glass dielectrics have been roughened with a roughness some orders smaller than the diameter of one filament. This roughness compensates for erosional effects the filaments may have on the dielectrics and so enhances the lifetime and reproducibility of the discharge. The discharge cell is 
mounted in a closed chamber. Since the experiments were performed without gas flow, the chamber was evacuated and refilled after each measurement.

All presented experiments are performed in pure helium at a pressure of $200 \mathrm{hPa}$. The discharge is electrically supplied with a function generator (HP 33120A) whose sinusoidal signal is amplified to voltage amplitudes of several hundred volts (AMT 0.6B100-LC). The amplifier limits the supply voltage frequency to about $100 \mathrm{kHz}$. The electrical behaviour is monitored with a digital oscilloscope (Yokogawa 700430, $150 \mathrm{MHz}$ ). The applied voltage amplitude $\hat{U}$ is measured via a voltage divider, the real current through the discharge is measured via a Wheatstone bridge. The lateral structure of the discharge is acquired with a Proxitronic image intensified camera that has a resolution of $512 \times 480 \mathrm{px}$. An exposure time of $200 \mu \mathrm{s}$ and a frame rate of $250 \mathrm{fps}$ were used. Because of the decreasing luminosity of the discharge at low frequencies, the lowest used frequency is limited by the sensitivity of the camera to $50 \mathrm{kHz}$. In the post processing, images are averaged over three camera frames to reduce noise, and inhomogenities in the camera sensitivity are equalised.

\section{The triple correlation function}

The triple correlation function (TCF) is used to evaluate the occuring patterns in the discharge. It can be applied to any structure that allows to determine reliable positions of the available substructures, e.g. filaments. In general, the structure must consist of solitary objects of any form. Unlike the two-dimensional fourier transformation, which is commonly used to analyse spatial patterns, the TCF ignores the geometry of the substructures and considers only the positions. The technique of TCF is already utilized in the field of dusty plasmas [13,14], but has to our knowlegde never been applied to patterned discharges. Its purpose is to analyze the filament positions and to estimate a probability distribution of finding a second filament at a certain angle and distance from a first filament. In this work, the filament position is defined as the centre of the filaments luminescence density.

As shown in Figure 2, the positions of any triple of filaments $(i, j, k)$ are considered. The interfilamentary distances $r^{\prime}=|\overline{j i}|$ and $r=|\overline{j k}|$ as well as the enclosed angle $\varphi=\operatorname{angle}(i, j, k)$ are determined. As we want to investigate a hexagonal lattice, we know that there is a typical nearest neighbour distance $d_{0}$ of filaments. Hence, the value of $r^{\prime}$ is limited to $r_{\max }^{\prime}$, which is chosen to be slightly larger than the typical neighbour distance $d_{0}$. Triples with $r^{\prime}$ larger than $r_{\max }^{\prime}$ are discarded, the other ones are sorted into bins with the dimensions $\Delta r$ and $\Delta \varphi$. The counts for these bins give the abundance of occurrence $n_{y}(r, \varphi)$.

Finally, the normalised abundance $y$ is computed as $n_{y}$ normalised to the radius $r$ :

$$
y(r, \varphi)=\frac{1}{r} n_{y}(r, \varphi) .
$$

The TCF can be regarded as an extension of the pair correlation function by the angular resolution. The pair

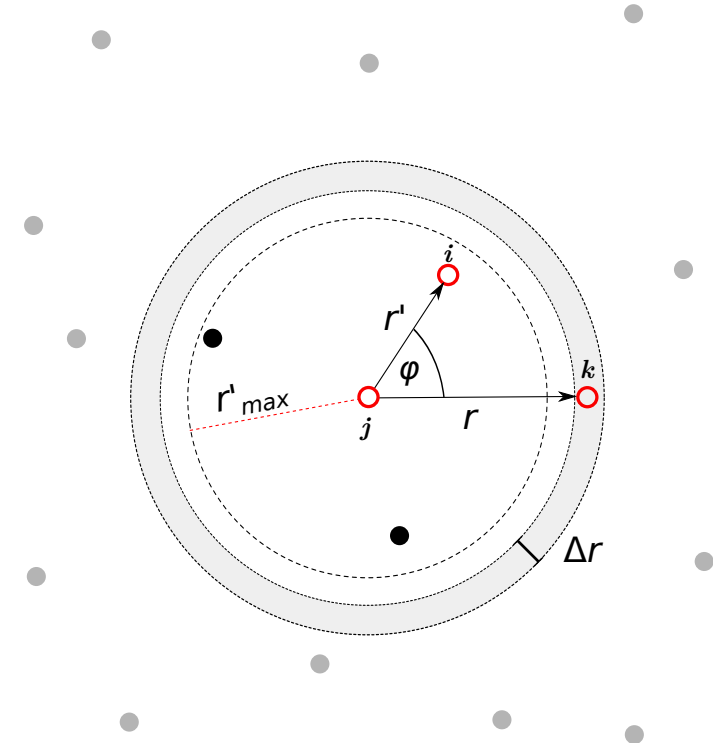

Fig. 2. (Color online) Representation of the triple correlation function. The distances $r^{\prime}, r$ and the angle $\varphi$ between any filament triples $(i, j, k)$ are calculated. $\circ-$ filaments used in current correlation, - - filaments considered for calculation of solely $r$ relative to $j, \bullet-$ filaments considered for calculation of both $r^{\prime}$ and $r$ relative to $j$.

correlation function has also been used to examine the arrangement of filaments in a BD [15], however, the additional angular resolution makes it easier to distinguish a rather strict order like a hexagonal lattice from other patterns.

\section{Hexagonal pattern breakdown at decreasing voltage}

Figure 3 exemplarily displays the pattern behaviour of the discharge at a frequency of $f=80 \mathrm{kHz}$ during voltage reduction. The amplitude of the applied voltage is decremented from $400 \mathrm{~V}$ to $300 \mathrm{~V}$. The maximum value is low enough to avoid parasitic arc discharges in areas other than the discharge gap, but sufficiently high to produce stable hexagonal discharge patterns. As the voltage is reduced, the pattern dissolves into a loose arrangement of filaments, before the discharge is eventually distinguished. The selected images were shot at the voltages $\hat{U}=363 \mathrm{~V}, 358 \mathrm{~V}, 350 \mathrm{~V}$, and $342 \mathrm{~V}$. First disturbances of the hexagonal order occur at $\hat{U} \approx 360 \mathrm{~V}$. Starting from the egde of the planar cell, growing movement of the filaments disturbs the order of the structure more and more. In the course of voltage reduction the number of filaments becomes reduced and at $\hat{U} \approx 345 \mathrm{~V}$ the filaments are almost completely randomly distributed.

The pattern series is examined with the $2 \mathrm{D}$ Fourier transformation (2D-FT) using the FFTW code described in [16]. The images in Figure 3 are set into the Fourier space via a fast Fourier transformation (Fig. 4, left-hand side). These two dimensional plots are referred to as 

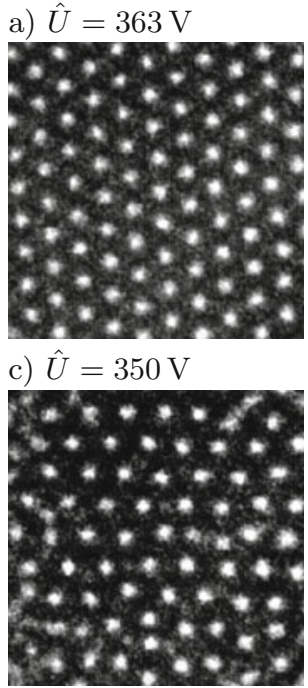

b) $\hat{U}=358 \mathrm{~V}$

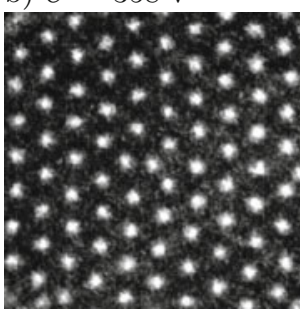

d) $\hat{U}=342 \mathrm{~V}$

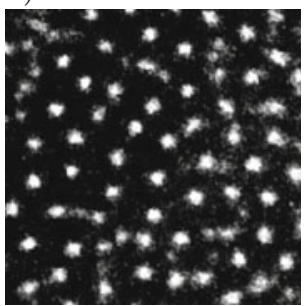

Fig. 3. (Color online) Luminescence pattern development during voltage reduction at various voltages. Other parameters: $f=80 \mathrm{kHz}, p=200 \mathrm{hPa}, d=0.5 \mathrm{~mm}$, no gas flow. The edge lengths of the depicted electrode surface is $31 \mathrm{~mm}$.

$\mathrm{FT}(\nu, \phi)$, whereas $\nu$ is the spatial frequency with its origin in the centre and $\phi$ is the polar angle. The displayed brightness of the diagram corresponds to the logarithmic amplitude of each Fourier component in arbitrary units.

For the highest voltage $(\hat{U}=363 \mathrm{~V})$, when the hexagonal pattern is completely intact, the well known corresponding Fourier transformation is seen. Upon voltage reduction, the sharp peaks in the $2 \mathrm{D}-\mathrm{FT}$ begin to dissolve. Finally, for the random filament distribution at $\hat{U}=342 \mathrm{~V}$, the FT becomes isotropic and the only radial structure is the wavelength defined by the filament diameter.

For a quantitative description of the pattern dissolution, an appropriate measure for the hexagonal order has to be introduced. Therefore, either the rotational or the translational symmetry of the pattern can be utilised. To quantify the translational symmetry, radial profiles $Y_{\text {rad }}$, i.e. integrated intensities around concentric circles with the 2D-FT origin as centre, are computed, with

$$
Y_{\text {rad }}(\nu)=\int_{0}^{2 \pi} \mathrm{d} \phi \operatorname{FT}(\nu, \phi) .
$$

Figure 5a shows the plots of $Y_{\text {rad }}(\nu)$ for the 2D-FTs from Figure 4. The first peak in such a plot corresponds to the fundamental frequency $\nu_{0}$, which is the inverse of the nearest neighbour distance $d_{0}=\nu_{0}^{-1}$. Its relative width can be used as a measure for the radial order. However, the data does not allow to determine the width in a reliable way. To quantify the azimuthal order a round profile is used, i.e. the amplitude along a circle at the fundamental frequency $\nu_{0}$, with

$$
Y_{\text {round }}(\phi)=\mathrm{FT}\left(\nu=\nu_{0}, \phi\right) \text {. }
$$

a)
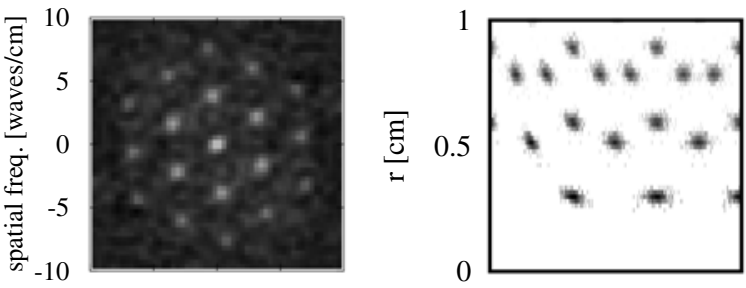

b)
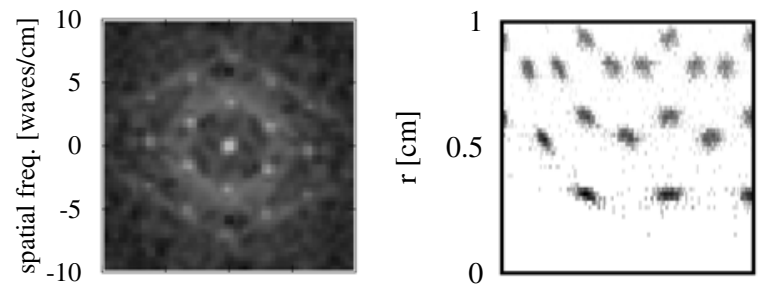

c)
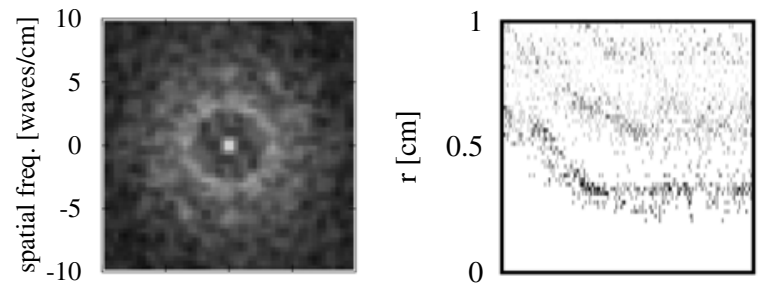

d)
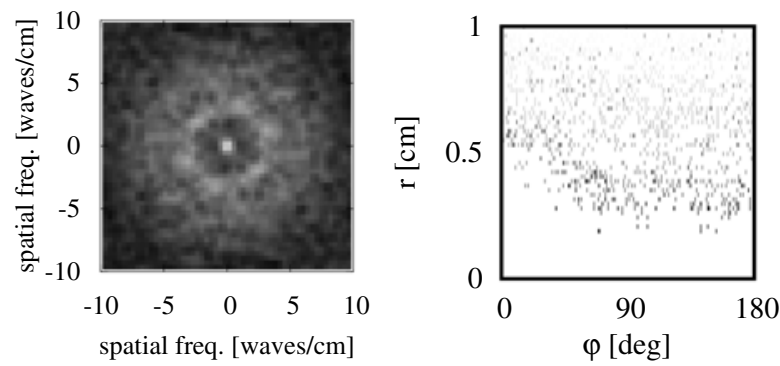

Fig. 4. (Color online) Line-up of the $2 \mathrm{D}$ Fourier transformation (left column) and triple correlation functions (right column) for the patterns depicted in Figure 3. (a) $\hat{U}=363 \mathrm{~V}$, (b) $\hat{U}=358 \mathrm{~V}$, (c) $\hat{U}=350 \mathrm{~V}$, (d) $\hat{U}=342 \mathrm{~V}$. The amplitudes of the 2D-FT are displayed in a logarithmic scale. For the TCF, the bin sizes are $\Delta r=0.18 \mathrm{~mm}$ and $\Delta \varphi=1^{\circ}, r_{\max }^{\prime}$ is $3.15 \mathrm{~mm}$.

Figure 5b depicts $Y_{\text {round }}(\phi)$ for various supply voltages. A perfect hexagon produces six equidistant peaks in such a plot. As the hexagonal pattern is still intact for higher voltages, the peaks are clearly observable. At lower voltages, the peak height is diminishing. This goes along with a breakdown of azimuthal order.

As a measure for the azimuthal order the ratio

$$
H_{\text {ang, FT }}=\frac{\max \left(Y_{\text {round }}(\phi)\right)}{\left\langle Y_{\text {round }}(\phi)\right\rangle_{\phi}}
$$

is defined. Figure 6 shows its dependence on the supply voltage. The plot shows a discontinuity in $H_{\text {ang, FT }}$ at $U \approx$ $350 \mathrm{~V}$. The large scattering in $H_{\mathrm{ang}}$, FT makes it difficult to tell whether the discontinuity is in the zeroth or first derivative of $H_{\text {ang, FT. }}$. 


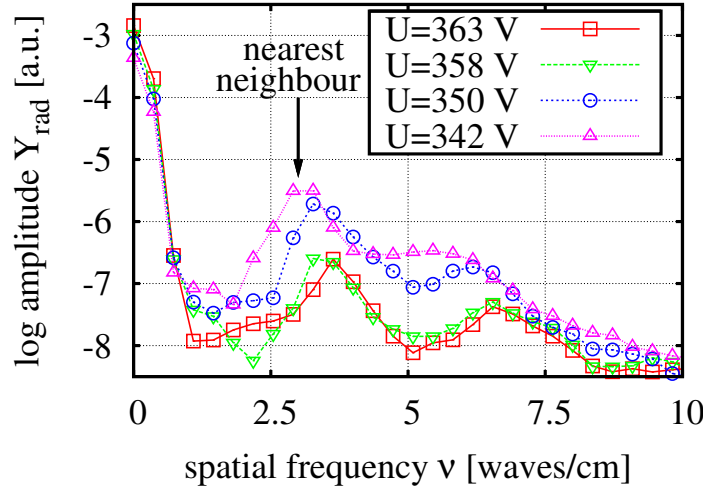

(a)

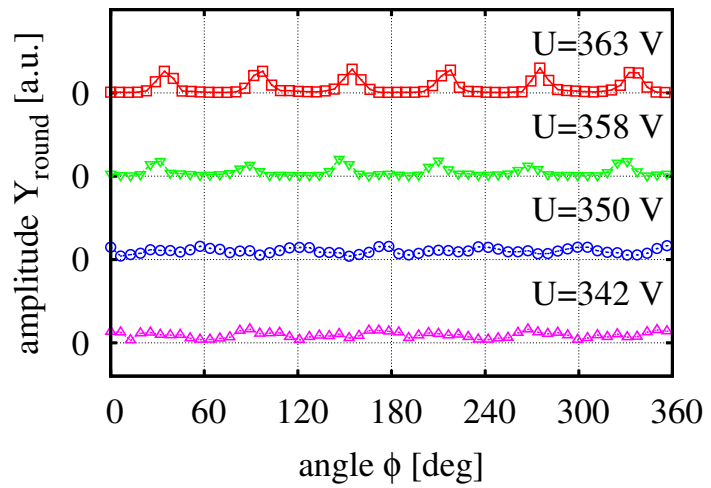

(b)

Fig. 5. (Color online) Analysis of the 2D Fourier transformation in Figure 4. (a) Azimuthally averaged radial profiles of 2D Fourier transformation at various voltages. (b) Round profile of $2 \mathrm{D}$ Fourier transformation at the respective radius of the first order peak for various voltages.

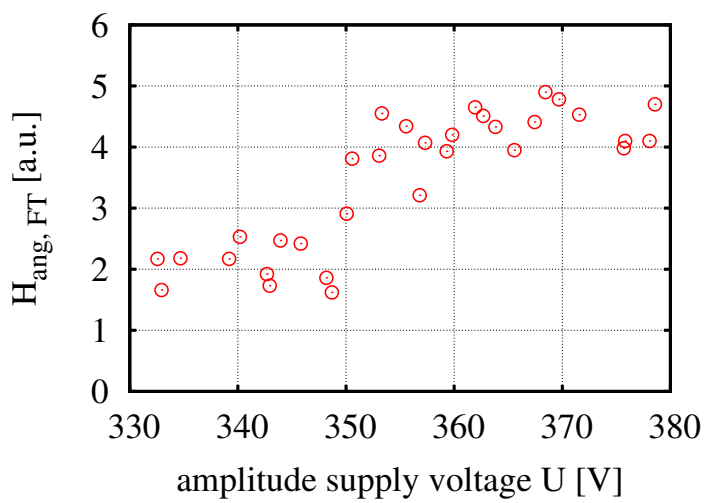

Fig. 6. (Color online) Ratio of the highest amplitude in the round profile to its mean value in dependence of the supply voltage.

The same transition is now investigated using the triple correlation function. In Figure 4, the TCF are shown next to their corresponding 2D-FT image. For distances below the distance of nearest neighbours $d_{0}$ (e.g. $d_{0}=3.15 \mathrm{~mm}$ in Fig. 4, TCF at $U=363 \mathrm{~V}$ ), the abundance is close to zero. The same lower limit for the distance of neighbouring filaments is also responsible for the nearly vanishing abundance at small angles. Physically, the lowest filament distance is determined by the inhibition of ignition in the vicinity of a filament $[17,18]$. Hence, the minimal filament distance is independent from the hexagonal pattern, and so the low abundance for small distances and small angles stays stable after the pattern breakdown (Fig. 4, TCF at $U=342 \mathrm{~V}$ ). This corresponds to the observed fundamental frequency $\nu_{0}$ in the radial profile of the 2D-FT, that apparently remains after the transition. For an intact hexagonal pattern (Fig. 4, TCF at $U=363 \mathrm{~V}$ ), the abundance at the distance $d_{0}$ has three peaks, namely at $60^{\circ}, 120^{\circ}$, and $180^{\circ}$. As the hexagonal pattern dissolves with decreasing supply voltage, the peaks get broader. At the end of this transition, the peaks have completely disappeared in azimuthal direction. In radial direction, only a small modulation stays visible.

Analogue to the 2D-FT interpretation, a measure for the hexagonal order shall be introduced. As the peak at $(r, \varphi)=\left(d_{0}, 60^{\circ}\right)$ is a well defined feature of the hexagonal pattern, its height $y_{60}=y\left(d_{0}, 60^{\circ}\right)$ is taken as the basis for the measure of order. To classify the angular order, $y_{60}$ relative to the average abundance at the fixed distance $d_{0}$ is calculated. Similarly, for the radial order $y_{60}$ relative to the average abundance at the fixed angle of $60^{\circ}$ is chosen. Hence, two quantities being a measure for the hexagonal order are defined:

$$
\begin{aligned}
& H_{\mathrm{ang}, \mathrm{TCF}}=\frac{y_{60}}{\left\langle y\left(d_{0}, \varphi\right)\right\rangle_{\varphi}} \\
& H_{\mathrm{rad}, \mathrm{TCF}}=\frac{y_{60}}{\left\langle y\left(r, 60^{\circ}\right)\right\rangle_{r}} .
\end{aligned}
$$

Figure 7 a shows the order analysis using $H_{\text {ang,TCF }}$ and $H_{\text {rad,TCF }}$ for the previously observed breakdown in Figures 3 and 4 . Basically, both plots display the same behaviour: for high voltages the measure of order remains nearly constant. For very low voltages (approximately $340 \mathrm{~V}$ in Fig. 7a), the peak $y_{60}$ vanishes in the noise. This is due to low order and additionally due to a decreased number of filaments (Fig. 7b). Hence, below a certain supply voltage $H_{\mathrm{ang}, \mathrm{TCF}}$ and $H_{\mathrm{rad}, \mathrm{TCF}}$ are not meaningful anymore.

The transition from a nearly constant $H_{\text {ang,TCF }}$ or $H_{\text {rad,TCF }}$ to a decreasing order is rather abrupt, so obviously there is a bifurcation from an ordered to an unordered system via a supercritical bifurcation. For a reliable computation of the bifurcation point $\hat{U}_{\mathrm{b}}$, the curves in Figure $7 \mathrm{a}$ are fitted with equation (7).

$$
q_{\mathrm{fit}}(\nu)=\left\{\begin{array}{l}
m \cdot \hat{U}+b \quad \hat{U}<\hat{U}_{\mathrm{b}} \\
m \cdot \hat{U}_{\mathrm{b}}+b \hat{U} \geq \hat{U}_{\mathrm{b}}
\end{array}\right.
$$

Here, $\hat{U}_{\mathrm{b}}$ depicts the bifurcation point, $m$ and $b$ are the slope and the offset of the straight line for $\hat{U}<\hat{U}_{\mathrm{b}}$. The bifurcation points in Figure $7 \mathrm{a}$ are $\hat{U}_{\mathrm{b}}$, ang $=362 \mathrm{~V}$ for the angular order and $\hat{U}_{\mathrm{b}, \mathrm{rad}}=356 \mathrm{~V}$ for the radial order. In all measurements, the bifurcation point $\hat{U}_{\mathrm{b} \text {, rad }}$ for the radial order is at lower voltages than the bifurcation point $\hat{U}_{\mathrm{b} \text {, ang }}$ for the angular order. 


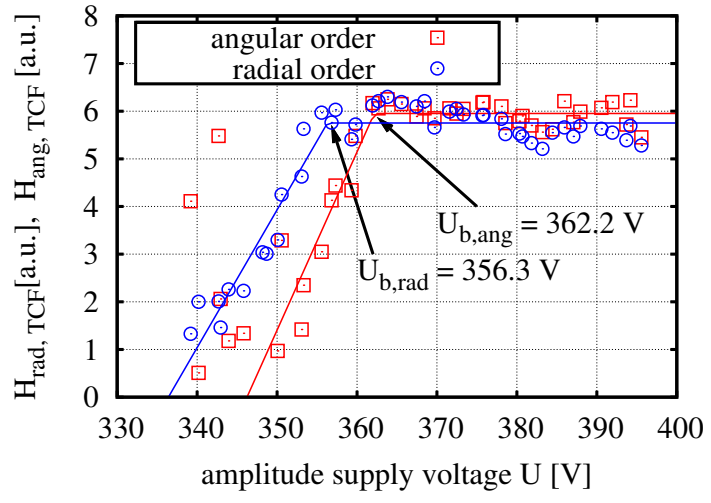

(a)

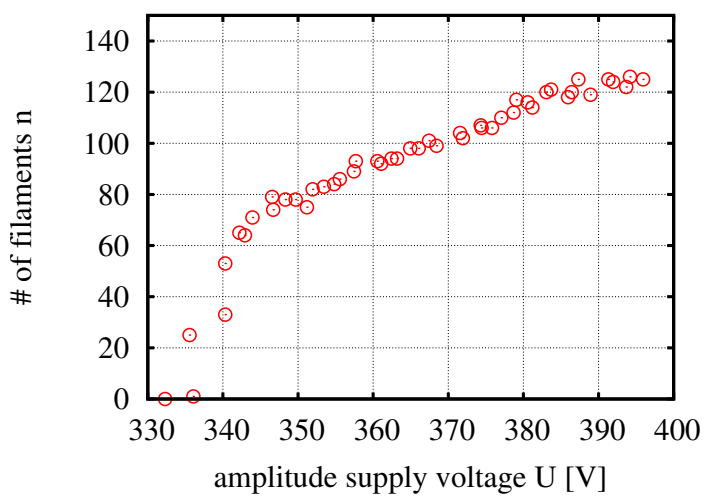

(b)

Fig. 7. (Color online) (a) Magnitude of angular and radial order corresponding to equations (5) and (6) as a function of the applied voltage amplitude. (b) Number of filaments as a function of the applied voltage amplitude.

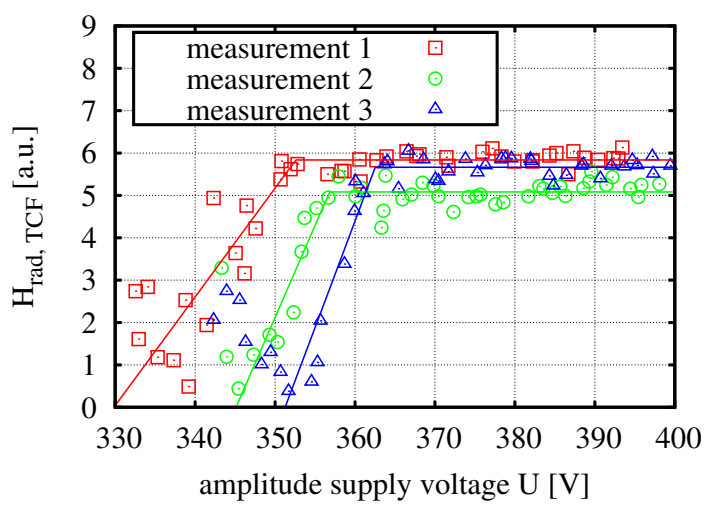

Fig. 8. (Color online) Scattering of the angular order bifurcation point with respect to the applied voltage. Parameters for all three measurements: $f=75 \mathrm{kHz}, p=200 \mathrm{hPa}, d=0.5 \mathrm{~mm}$, no gas flow.

However, the absolute bifurcation point may vary. Figure 8 shows exemplarily three independent bifurcation measurements for the angular order that were each done with the same experimental conditions, $f=75 \mathrm{kHz}$, $p=200 \mathrm{hPa}, d=0.5 \mathrm{~mm}$ and without gas flow. While the bifurcation was reproduced, the actual value for the bifurcation voltage varies between $350 \mathrm{~V}$ and $365 \mathrm{~V}$.
A correlation between the applied frequency and the bifurcation voltage could not be found. If there is a dependence between those quantities, it is camouflaged by the bifurcation voltage uncertainty.

\section{Conclusion}

The hexagonal pattern breakdown in self-organised dielectric barrier discharges has been investigated with the $2 \mathrm{D}$ Fourier analysis technique and for the first time with the TCF. While decreasing the supply voltage of the discharge system, the lateral pattern changes from a regular hexagonal filament arrangement to an unordered arrangement of filaments. The loss of order is observable both in the 2DFT and in the TCF. However, the definition of a scalar measure of order is succesful only for the TCF. For the 2D$\mathrm{FT}$, the measure for lateral order suffers from the width and the overlap of neighbouring maxima. A measure for the angular order is determinable, but its values scatter rather wide, which makes the determination of a bifurcation type impossible. In contrast, the measures of order derived from the TCF, $H_{\text {rad,TCF }}$ and $H_{\text {ang,TCF}}$, both show a distinct bifurcation point within a supercritical bifurcation.

To understand this discrepancy, it is necessary to regard the mechanism of filament interaction. Different experiments indicate that the filament interaction is best described by a particle-like approach, i.e. the interaction takes place on the basis of direct neighbourhood [18,19], especially in the regime of loose filament arrangements [11]. This results in a strong local order but does not enforce a long-range order.

The Fourier transformation is a global approach of a pattern description. To rate the lateral order of a hexagonal pattern, the width of the peaks at the fundamental frequency $\nu_{0}=d_{0}^{-1}$ should be determined. However, from the actual data, this is not possible in a reliable way. The derived measure for the angular order reveals a distinct bifurcation between an ordered and unordered filament arrangement, but it suffers from long-range deviations in the pattern during the transition phase which blur the otherwise sharp bifurcation point.

On the other hand, the TCF emphasises the local arrangement of filaments. Thus, the derived measures of order $H_{\text {rad, TCF }}$ and $H_{\text {ang, TCF }}$ both are able to describe the transition between the states of order well and to determine a well defined bifurcation point.

The analysis of several measurements leads to the conclusion, that the bifurcation voltage cannot exactly be reproduced, as it varies in the intervall between $350 \mathrm{~V}$ and $365 \mathrm{~V}$. The sequence of the breakdown of order, i.e. angular order at higher voltages and radial order at lower voltages, however, is not affected. Further, a possible dependence between applied frequency and bifurcation voltage has not been found in the course of this investigation.

In order to discuss the transition from a physical point of view, one has to bear in mind that it occurs close to the ignition voltage of the discharge. Above the ignition voltage, the discharge is ignited everywhere, where the 
inhibitory interaction of a neighbouring filament is negligible, since the applied voltage is sufficient. This would result in a discharge pattern with the highest packing density, hence a hexagonal pattern. Reducing the voltage below ignition voltage results in a pattern that consists only of the filaments that are maintained by residual surface charges, reignition of new filaments in unoccupied places can no longer occur. Thus, filaments may arrange randomly with an arbitrarily large inter-filament distance. This is consistent with the observed behaviour below the bifurcation voltage.

This work has been funded by the Deutsche Forschungsgemeinschaft, SFB-TRR 24, B14. We also acknowledge the supportive work of Peter Druckrey, Uwe Meißner and Thomas Schumann. We would like to thank André Schella and André Melzer for the hint at the TCF and fruitful discussions.

\section{References}

1. U. Kogelschatz, Plasma Chem. Plasma Process. 23, 1 (2003)

2. H.-E. Wagner, R. Brandenburg, K.V. Kozlov, A. Sonnenfeld, P. Michel, J.F. Behnke, Vacuum 71, 417 (2003)

3. J.-P. Boeuf, J. Phys. D 36, R53 (2003)

4. G. Fridman, G. Friedman, A. Gutsol, A.B. Shekhter, V.N. Vasilets, A. Fridman, Plasma Process. Polym. 5, 503 (2008)

5. K.-D. Weltmann, E. Kindel, T. von Woedtke, M. Hähnel, M. Stieber, R. Brandenburg, Pure Appl. Chem. 82, 1223 (2010)
6. W. Breazeal, K.M. Flynn, E.G. Gwinn, Phys. Rev. E 52, 1503 (1995)

7. A.L. Zanin, E.L. Gurevich, A.S. Moskalenko, H.U. Bödeker, H.-G. Purwins, Phys. Rev. E 70, 036202 (2004)

8. L.F. Dong, Z. Yin, L. Wang, G. Fu, Y. He, Z. Chai, X. Li, Thin Solid Films 435, 120 (2003)

9. E.L. Gurevich, A.L. Zanin, A.S. Moskalenko, H.-G. Purwins, Phys. Rev. Lett. 91, 154501 (2003)

10. L.F. Dong, H. Yue, Y.F. He, W.L. Fan, H. Xiao, J.Y. Chen, Z.G. Bai, Phys. Plasmas 17, 082302 (2010)

11. L. Stollenwerk, S.V. Gurevich, J.G. Laven, H.-G. Purwins, Eur. Phys. J. D 42, 273 (2007)

12. L. Stollenwerk, S. Amiranashvili, J.-P. Boeuf, H.-G. Purwins, Eur. Phys. J. D 44, 133 (2007)

13. P. Ludwig, H. Thomsen, K. Balzer, A. Filinov, M. Bonitz, Plasma Phys. Control Fusion 52, 124013 (2010)

14. A. Schella, T. Miksch, A. Melzer, J. Schablinski, D. Block, A. Piel, H. Thomsen, P. Ludwig, M. Bonitz, Phys. Rev. E 84, $056402(2011)$

15. A. Chirokov, A. Gutsol, A. Fridman, K.D. Sieber, J.M. Grace, K.S. Robinson, Plasma Sources Sci. Technol. 13, $623(2004)$

16. M. Frigo, S.G. Johnson, Proc. IEEE 2, 216 (2005)

17. L. Stollenwerk, H.-G. Purwins, Contrib. Plasma Phys. 70, $22(2005)$

18. L. Stollenwerk, New J. Phys. 11, 103034 (2009)

19. I. Brauer, M. Bode, E. Ammelt, H.-G. Purwins, Phys. Rev. Lett. 84, 4104 (2000)

Open Access This is an open access article distributed under the terms of the Creative Commons Attribution License (http://creativecommons.org/licenses/by/3.0), which permits unrestricted use, distribution, and reproduction in any medium, provided the original work is properly cited. 